\title{
Gas Sensitivity of the surface potential of Pyrene Coated ZnO Nanorods
}

\author{
Surya Elangovan ${ }^{1}$, Surya Velappa Jayaraman ${ }^{1,2}$ Gabriele Magna $^{3}$, \\ Eugenio Martinelli ${ }^{3}$, Roberto Paolesse ${ }^{4}$, Corrado Di Natale ${ }^{3^{*}}$, Yuvaraj Sivalingam $^{1,2^{*}}$ \\ ${ }^{1}$ Department of Physics and Nanotechnology, SRM Institute of Science and Technology, \\ Kattankulathur, Kanchipuram - 603203, Tamil Nadu, India \\ ${ }^{2}$ SRM Research Institute, SRM Institute of Science and Technology, Kattankulathur, Kanchipuram - \\ 603203, Tamil Nadu, India \\ ${ }^{3}$ Department of Electronics Engineering, University of Rome Tor Vergata, Via del Politecnico 1, Roma \\ - 00133, Italy \\ ${ }^{4}$ Department of Chemical Science and Technology, University of Rome Tor Vergata, Via del \\ Politecnico 1, 00133 Roma, Italy \\ Corresponding author's e-mail address: magna.gabriele@gmail.com
}

\begin{abstract}
Organically capped metal oxide nanostructures offer an interesting combination of chemical and physical sensitivities. In particular, the sensitivity to light and to gases have been shown to cooperate in order to strength each other. In this paper, we studied the properties of pyrene tetratopic ligands (PTL) and $\mathrm{ZnO}$ nanorods. After structural and morphological characterizations of the nanohybrid materials, mutual cooperation of selectivity to visible light and to volatile organic compounds (VOCs) is investigated measuring the surface potential distribution with a scanning kelvin probe. Results show the photo assisted selective response to donor compounds such as triethylamine.
\end{abstract}

Key words: ZnO nanorods, Pyrene tetratopic ligands, Contact potential difference, Surface photovoltage, Gas adsorption.

\section{Introduction}

Organic/inorganic hybrid nanomaterials have been widely studied for optoelectronic applications and also as photo-enhanced gas sensors [1]. To improve the performance of sensors, it is of outmost importance to understand the properties of the organicinorganic interfaces. Scanning kelvin probe (SKP) is one of the most effective tools to study interfaces. In this work, pure and PTL coated $\mathrm{ZnO}$ nanorods are synthesized [2] and their behaviour under gas adsorption is examined using SKP

\section{Experimental}

ZnO nanorods were synthesized with a hydrothermal procedure. For the scope, a seed layer was deposited by sputtering on clean $\mathrm{p}$ type silicon substrates. Seed layer coated substrates were then immersed in a precursor solution made of $0.025 \mathrm{~mol} / \mathrm{L}$ of Zinc acetate dehydrate and hexamethyltetramine and kept at $90^{\circ} \mathrm{C}$ for 4 hours [1].
As grown $\mathrm{ZnO}$ nanorods were finally functionalized with PTL by drop casting (Fig.1a). The prepared materials were duly characterized investigating morphological, and optical properties. Surface potential was measured to characterize the response of the materials to the exposure to both VOCs and light. For the scope a SKP machine equipped with a surface photovoltage module (SKP5050 from KP Technology Ltd., UK) was used.

The Kelvin probe is a capacitor made by a gold tip and the substrate with the air as dielectric (Fig. 1b). The gold tip in the used SKP, was kept in vibration at a frequency of about $78 \mathrm{~Hz}$. At the equilibrium the capacitor is charged at the contact potential difference (CPD) which is the difference of the work function of the gold and the sample [3]. Assuming that the work function of the gold tip does not change, any change of the CPD is attributed to a change of the surface potential of the sample.

All measurements were performed at room temperature and in ambient conditions using a 
Au tip with a diameter of $2 \mathrm{~mm}$. To investigate the relative variation of the CPD of as grown and PTL coated $\mathrm{ZnO}$ samples, the gold tip was scanned across the sample surface $\sim 3 \mathrm{~mm}^{2}$ area (Raster scan) in dark and under visible light illumination.

The samples were exposed to vapors of ethanol, n-hexane and triethylamine.

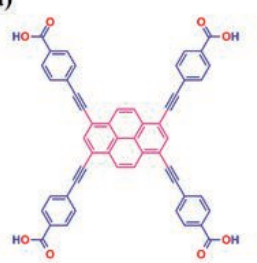

(b)

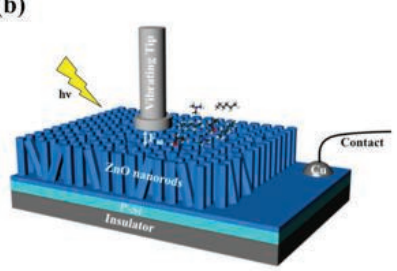

Fig. 1. (a) Molecular structure of PTL, (b) Schematic illustration of surface photovoltage measurements of as grown and PTL coated $\mathrm{ZnO}$ nanorods using scanning kelvin probe system.

\section{Results and Discussion}

Fig.2a shows the FESEM image of PTL coated $\mathrm{ZnO}$ nanorods. The diameter of the nanorods is ine the ran125 nm - $332 \mathrm{~nm}$ while the length is in the intervl $4.1 \mu \mathrm{m}-4.6 \mu \mathrm{m}$. Hexagonal face cut suggests the wurtzite structure. Fig. $2 b$ shows the corresponding TEM image.

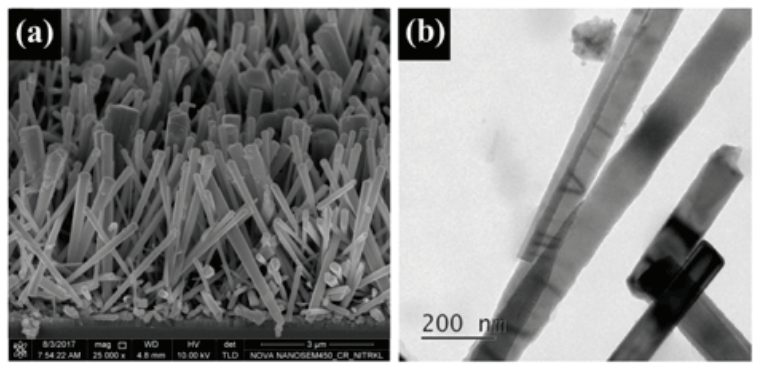

Fig. 2. Morphological characterization of PTL functionalized $\mathrm{ZnO}$ nanorods. (a) $45^{\circ}$ tilted FESEM image and (b) TEM image.
Fig. 3a shows the time evolution of the CPD under white light illumination while the samples are kept in a n-hexane atmosphere. Fig. 3b shows the same measurement but in a triethylamine atmosphere. In Flg. 4 the corresponding photovoltage defined as the CPD shift du to illumination are compared. The interaction between photosensitivity and gas sensitivity is opposite for the two VOCs. This behavior can be explained considering that PTL absorbs visible light and then, under light, photo induced electrons are transferred from PTL to $\mathrm{ZnO}$ surface. This creates a number of free electron states in PTL that can be conveniently filled by electrons from donor species such as triethylamine. The opposite behavior under exposure to $\mathrm{n}$-hexane can be explained considering the dispersion interaction between n-hexane and the PTL layer. N-hexane molecules intercalate in the PTL layer hindering the electron transfer from PTL to $\mathrm{ZnO}$.

\section{Conclusion}

These results show that the photo response of PTL capped $\mathrm{ZnO}$ nanostructure can be tuned by suitable organic molecules functionalization. This study is useful for developing a new generation of self-powered gas sensors.

\section{References}

[1] Y. Sivalingam, E. Martinelli, A. Catini, G. Magna, G. Pomarico, F. Basoli, R. Paolesse, C. Di Natale, J. Phys. Chem. C. 116 (2012) 91519157. doi:10.1021/jp302225u.

[2] Y. Sivalingam, P. Elumalai, S.V.J. Yuvaraj, G. Magna, V.J. Sowmya, R. Paolesse, K.-W. Chi, Y. Kawazoe, C. Di Natale, Journal of Photochemistry and Photobiology A: Chemistry. 324 (2016) 62-69. doi:10.1016/j.jphotochem.2016.02.023.

[3] J.R. Harwell, T.K. Baikie, I.D. Baikie, J.L. Payne, C. Ni, J.T.S. Irvine, G.A. Turnbull, I.D.W. Samuel, Phys. Chem. Chem. Phys. 18 (2016) 1973819745. doi:10.1039/C6CP02446G.
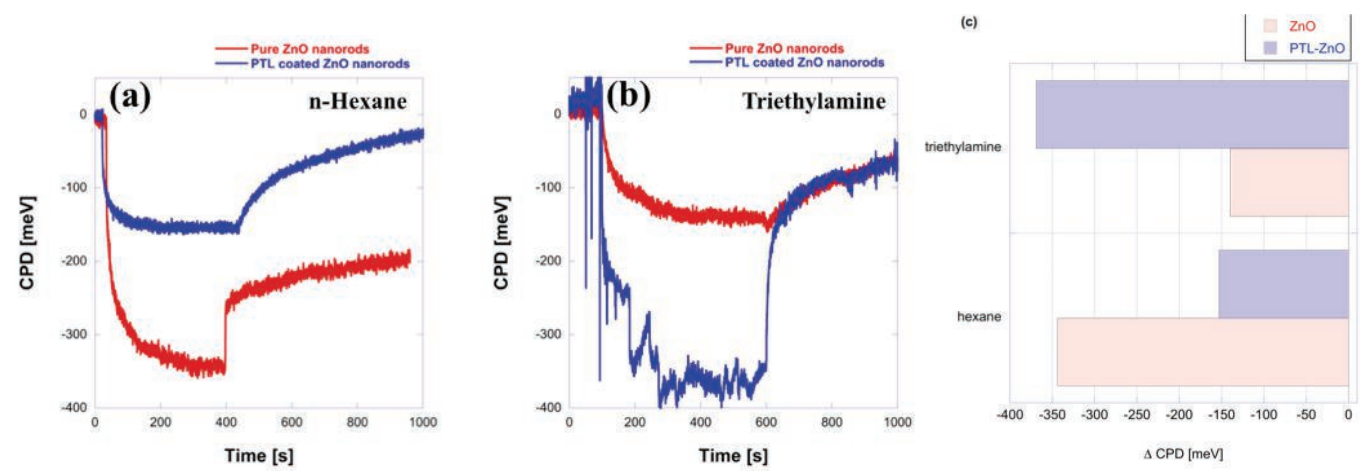

Fig. 3. CPD change due to illumination. (a) in n-hexane atmosphere, (b) in triethylamine atmosphere. (c) comparison of the equilibrium photovoltage signal. 